
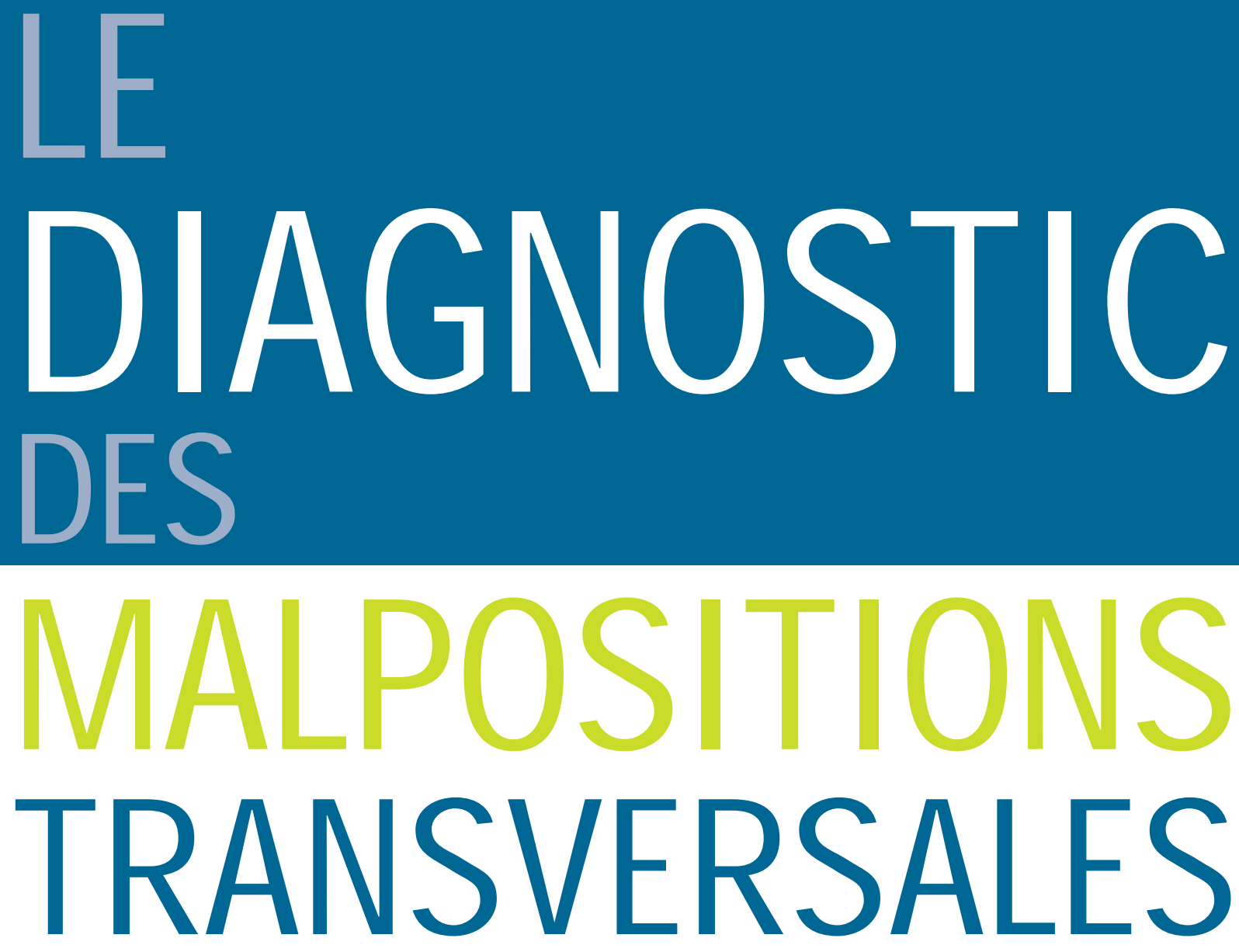

\title{
Georges BERNADAT
}




\section{INTÉRÊT}

es moulages dentaires et la téléradiographie de profil sont deux documents scientifiques à la base de l'évolution de la discipline orthodonIA TROSSLEm tique. Mais, tant la classifiDIMENSION, TRANSUERSAL, EST IE PARENT PAUURE DES PUBLIBATIONS cation d'ANGLE, proposée à l'examen des moulages, que les multiples analyses céphalométriques qui jalonnent l'histoire de l'orthodontie n'ont pris en compte

que les deux dimensions antéro-postérieure et verticale de l'espace. La troisième dimension, transversale, est le parent pauvre des publications. Ce fut le thème du volume 29 en 1995 de la revue d'ODF. Les articles de RICKETTS et de P. VION sur les clichés tridimensionnels qui y figurent, s'ils restent une référence scientifique, ne présentent plus aujourd'hui, pour nous praticiens, d'intérêt diagnostic en dehors des dissymétries basales qui sont hors de notre accès thérapeutique.

Nous démontrons régulièrement grâce à un montage dynamique que, si l'ATM peut supporter une légère adaptation antérieure, son adaptation transversale est impossible. Plus les contacts inter-arcades présenteront des prématurités transversales et plus celles-ci seront postérieures, plus le potentiel pathogène sera élevé.

Les excès maxillaires transversaux sont rares, les insuffisances sont très fréquentes. Ces dernières sont à l'origine des contacts prématurés et des interférences les plus pathogènes. La plupart du temps les endomaxillies sont le résultat d'une croissance soumise à une ventilation orale et à une position basse de la langue.

De même que le chirurgien-dentiste doit mettre en œuvre les moyens connus pour dépister la carie dentaire ou les pathologies cancéreuses au cours de sa consultation, il doit, de la même façon, s'assurer qu'un enfant ventile bien par le nez le jour et la nuit.

Le non dépistage de cette dysfonction doit devenir une faute professionnelle pour toute la famille médicale, en particulier pour le chirurgien-dentiste, le médecin référent de la famille, le pédiatre et l'ORL. Les conséquences néfastes d'une ventilation orale sur l'avenir physique et neurologique d'un enfant sont telles qu'il s'agit à notre avis d'une priorité de prévention. La pollution de l'air ne peut que continuer à aggraver la situation dans l'avenir.

Nous ne devons donc pas avoir peur de le dire clairement, haut et fort pour le bien de la santé de nos enfants. Notre énergie a été trop mobilisée et dispersée par des propositions mécaniques au détriment des fondamentaux de la croissance.

Comme nous l'avons vu dans un précédent article, la ventilation orale et la dysfonction linguale dégradent fortement l'harmonie de la croissance maxillaire et l'un des premiers gestes à réaliser est le rétablissement de sa dimension transversale (bulletin $n^{\circ} 36$ de juin 2008).

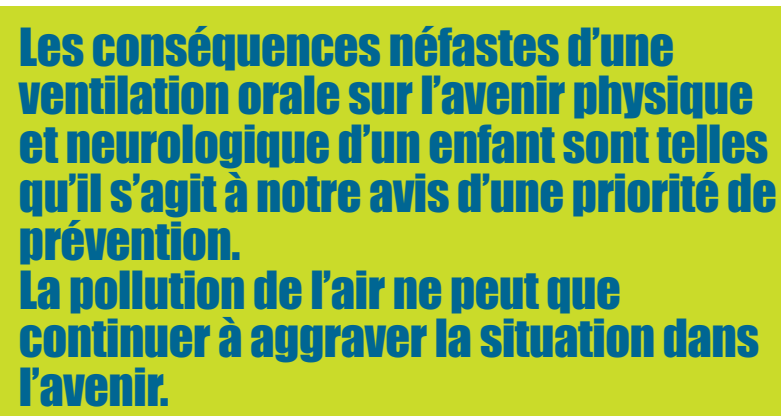




\section{LES MOTEURS DE LA GROISSANGE MAYULARE}

Il nous paraît utile d'essayer de faire ici l'inventaire des moteurs de la croissance maxillaire qui peuvent être nos alliés lorsqu'ils fonctionnent bien ou nos ennemis lorsqu'ils s'arrêtent ou sont déficients.

\subsection{L'ADN}

Ses anomalies entraînent la trisomie 21, les agénésies, les fentes vélo-palatines et certaines promandibulies qui ne sont que la partie visible de l'iceberg. Ces quelques anomalies ont été étudiées par de nombreux auteurs dans le passé, mais la connaissance de ces facteurs génétiques devrait s'élargir beaucoup avec les acquisitions scientifiques récentes. En fait, la sélection naturelle, chère à DARWIN, élimine depuis très longtemps les nourrissons des régions trop froides ou trop chaudes de notre planète s'ils ne peuvent ventiler par le nez. Les uns gèlent leurs poumons, les autres ne peuvent réguler la température de leur cerveau en cas d'infection ou de paludisme. Les hommes de ces régions ont donc évolué vers des nez et des faces larges. Seuls les hommes qui ont évolué dans des régions tempérées ont vu leur descendance survivre malgré des ventilations orales. Les américains d'origine ont été surpris par les « longs nez » blancs qui débarquaient de leurs bateaux. La croissance cartilagineuse, en particulier celle de la cloison médiane des fosses nasales est directement sous la dépendance de l'ADN. C'est une croissance en tache d'huile qui a une influence directe sur celle du nez, du prémaxillaire et de la dimension verticale de la région antérieure des fosses nasales. Le nez est un héritage familial, il est indispensable de l'évaluer dans le profil et de pronostiquer sa croissance.

\subsection{Le tissu nerveux}

Les trois cerveaux, reptilien, mamalien et humain vont s'additionner pour donner le crâne que nous connaissons aujourd'hui. La base du crâne, l'orientation de ses différentes pièces osseuses, si bien démontrée par les travaux de M. J. DESHAYES, sous les tensions des muscles de la posture, ont une influence mécanique directe sur le développement de la face. La continuelle formation de connexions neuronales modifie en permanence les comportements musculaires sous la dépendance directe de la proprioception, des apprentissages et du psychisme.

\subsection{La dynamique des fluides}

Les travaux de Jacques et Claude TALMANT sont fondamentaux dans ce domaine. Pendant la vie embryonnaire et après la naissance, la ventilation amniotique puis aérienne va développer les voies aériennes supérieures et organiser la pneumatisation des os (sinus frontaux, ethmoïdaux, sphénoïdaux et maxillaires). La déglutition amniotique puis salivaire, obéissant aux même lois, va aboutir à la formation de l'oro-pharynx.

\subsection{Les organes des sens}

Leurs récepteurs qui conditionnent la survie aux dangers sont très proches du cerveau pour des raisons de temps de conduction. Ceux de l'odorat, du goût, de la vision vont développer leurs propres volumes et influencer mécaniquement directement leur environnement proche. Les « expériences naturelles » décrites par J. DELAIRE ont apporté les preuves de cette influence mécanique.

\subsection{Le tissu musculaire et son comportement indissociable}

Les muscles élévateurs, propulseurs et abaisseurs de la mandibule, de la langue, de la posture et de la mimique par leur tonus au repos et leur activité au cours de la mastication, du stress, de la ventilation orale, de la déglutition, des tics et habitudes vont sculpter le squelette par lequel ils donnent les mouvements et les fonctions. De nombreux auteurs ayant publié sur la croissance ont relevé tel ou tel effet du comportement neuro-musculaire. Nous devons évoquer ici les différentes «membranes » comme l'épiderme, le derme et les aponévroses qui constituent une enveloppe élastique, source de contraintes passives.

Parmi tous ces comportements neuromusculaires, celui de la langue est une des constantes des dysfonctions. S'il s'agit pour nous d'une évidence, l'enfant doit absolument 
comprendre son importance et partager cette préoccupation avec nous. Il ne faut pas avoir peur de se mettre à sa portée. Nous avions l'habitude de comparer la bouche à la maison où doit habiter la langue. La voûte palatine en est le toit et les dents en sont les murs. La langue se repose la plupart du temps, même chez les grands bavards! La chambre est au premier étage sous le toit. C'est là qu'elle doit se reposer. Ce faisant, elle soutient le toit qui s'effondrerait sans elle. C'est bien ce qui se passe si elle reste au rez-de-chaussée ou au sous-sol. Elle ne doit pas non plus pousser les murs qui vont se déformer au risque de s'écrouler.

Dans un langage plus académique, n'oublions pas que la langue est constituée de 17 muscles qui ont chacun leur activité propre. Le simple calcul permet de comprendre que le nombre des combinaisons de ces différentes activités est très élevé. Les positions de la langue sont donc extrêmement variées. Comme les muscles sont des volumes hydriques ils sont certes déformables mais incompressibles. La première conséquence est que le volume lingual, accompagné de la pression qu'il engendre, qui quitte une zone va obligatoirement en occuper une autre. C'est bien ce que nous observons cliniquement. Lorsque la langue quitte la région palatine, elle descend dans la cavité buccale et l'équilibre des pressions avec les buccinateurs dans les secteurs latéraux disparaît. C'est ce déséquilibre des pressions qui provoque les nombreuses dysmorphoses transversales basales et alvéolaires que nous allons décrire. Le ramus et l'angle mandibulaires nous sont donnés par le comportement des muscles élévateurs, mais le corpus nous est donné par la langue. Nous n'aurons pas d'autre choix thérapeutique que d'adapter l'arcade maxillaire à l'arcade mandibulaire. C'est pourquoi l'analyse de l'encombrement dentaire mandibulaire est prioritaire sur celle de l'encombrement maxillaire.

\subsection{Le tissu osseux}

Sa croissance est sous la dépendance du périoste, lui-même sous celle des tractions ou pressions des muscles et tendons. Mais elle est aussi un paramètre d'ajustage au niveau de toutes les sutures. Grâce à cette fonction adaptative des sutures, les 7 sutures du maxillaire permettront presque toujours à la mandibule de retrouver une bonne fonction masticatoire. Nous avons obtenu des disjonctions non chirurgicales jusqu'à 28 ans, bien au delà de la fin de croissance.

\subsection{Les tissus dentaires}

L'os alvéolaire naît, vit et disparaît avec les dents. L'évolution des dents est créatrice d'os. C'est pourquoi nous avons abandonné l'idée de pratiquer des extractions précoces en dehors des DDM confirmées. D'autre part le mouvement orthodontique provoqué par des forces légères est lui-même créateur d'os.

\subsection{Le temps}

Il agit par l'intermédiaire des sécrétions hormonales. L'hypophyse n'a pas une action continue mais variable avec l'âge. Son dysfonctionnement peut être d'origine génétique ou tumorale, c'est une cause des très rares excès de croissance observés.

Les défauts de croissance sont la règle dans l'origine des dysmorphoses.

En effet l'arrêt ou le ralentissement d'un ou plusieurs de ces moteurs de croissance aboutit presque toujours à un défaut de croissance. Dans les trois sens de l'espace nous aurons donc des défauts verticaux, antéro-postérieurs ou transversaux. Nous nous intéresserons seulement à ces derniers dans cet article, bien que leurs interactions soient certaines car le maxillaire supérieur est bien un volume.

Nous ne pourrons hélas pas redémarrer le moteur génétique. La trisomie 21 ou l'acromégalie ne peuvent avoir, actuellement, qu'une réponse chirurgicale chez l'adulte. Dans l'article précédant, déjà cité, nous avons vu la chronologie d'un traitement interceptif et nous avons décrit les techniques de remise en route des moteurs fonctionnels. Le traitement a cependant commencé par la correction du défaut transversal.

Nous allons préciser ici comment diagnostiquer avec précision les défauts transversaux et planifier cette expansion quantitativement. 


\section{LES DIFFÉRENTES EXPRESSIONS DU DÉFAUT TRANSUERSAL}

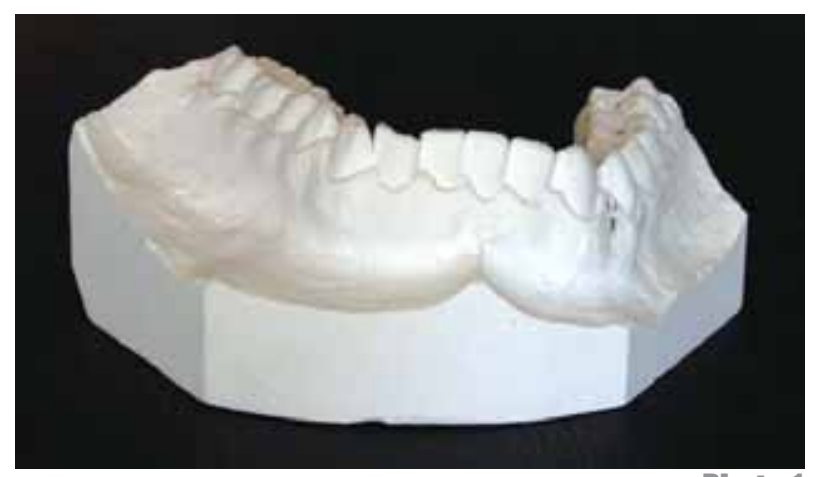

Photo 1

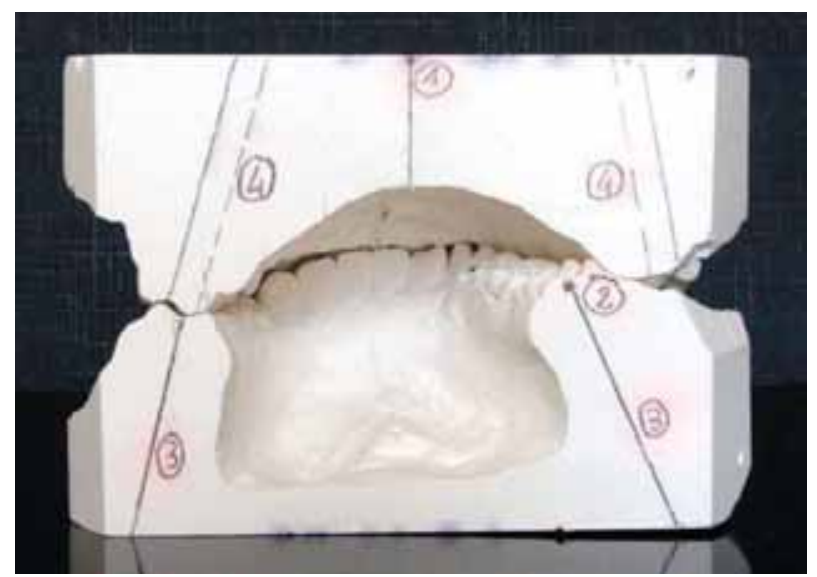

Photo 2

Les modèles en plâtre des arcades maxillaire et mandibulaire sont un document scientifique qui conserve toute sa valeur, en particulier parce qu'il est le document 3D le plus facile à obtenir et ce, sans irradiation du petit patient. Les renseignements qu'ils peuvent donner ont été sous-estimés à cause des exigences plus esthétiques que scientifiques de la taille " américaine ». Il convient, à notre avis, de préparer ces modèles avec l'objectif d'en tirer le maximum de renseignements fiables.

\subsection{La préparation des modlèles en plâtre}

Elle commence au cours de la prise d'empreintes. La partie endobuccale d'une plaque de FOX sera introduite en bouche et appliquée sur l'arcade maxillaire. Si la partie externe n'est pas parallèle avec le plan passant par les porions et les points orbitaires inférieurs et la ligne bipupillaire, un petit boudin de silicone sera interposé entre la plaque et la face occlusale de l'arcade pour obtenir ce parallélisme. Ce boudin de silicone sera utilisé au moment de la taille des modèles pour paralléliser le socle du modèle maxillaire avec les plans de référence. Bien que ce ne soit pas notre sujet, signalons au passage que les modèles étant posés sur une table horizontale, cette précaution préalable permet de visualiser instantanément les anomalies verticales (bascules du plan d'occlusion, égressions ou ingressions, photo 1). La cire ou le silicone mordu en OC permettra de paralléliser la base $\mathrm{du}$ modèle mandibulaire à celle du modèle maxillaire. Les dos des modèles seront ensuite parfaitement taillés ensemble en OC. Le dos du modèle mandibulaire taillé en premier parallèlement aux faces mésiales des premières molaires, si elles n'ont pas subi de mésioversion, servira alors de référence. Il nous paraît souhaitable aussi de paralléliser les tailles sur les côtés avec les gouttières occlusales des secteurs latéraux. La taille des angles postérieurs ne doit pas dépasser le collet vestibulaire de la dent la plus vestibulaire.

\subsection{Le traçage des modièles}

Une suite de points, tracés au crayon, repère parfaitement la suture inter-maxillaire depuis la papille inter-incisive jusqu'à la suture interpalatine. Une ligne sera tracée sur le dos du modèle maxillaire, perpendiculaire au socle, dans l'alignement de ces points (photo 2, ligne 1). Les excès de plâtre seront retirés dans la zone rétro-molaire. Les sommets des cuspides mésio-palatines des 16 et 26 et le fond des fosses des 36 et 46 seront aussi marqués au crayon. Ce sont les points $n^{\circ} 3$ des groupes III (figure 1). Avec l'aide d'une équerre plaquée sur le dos des modèles ces points seront reportés sur l'angle formé par le dos des modèles et le sommet de la gencive rétro-molaire (photo 2, point 2). La même démarche est possible avec les points no 5 situés sur les deuxièmes molaires vues en coupe grâce à l'artifice des modèles surtaillés de la photo 2 .

C'est par un examen attentif de la face postérieure des modèles en plâtre que nous 
GROUPE III ET POINTS CORRESPONDANTS À LA MANDIBULE

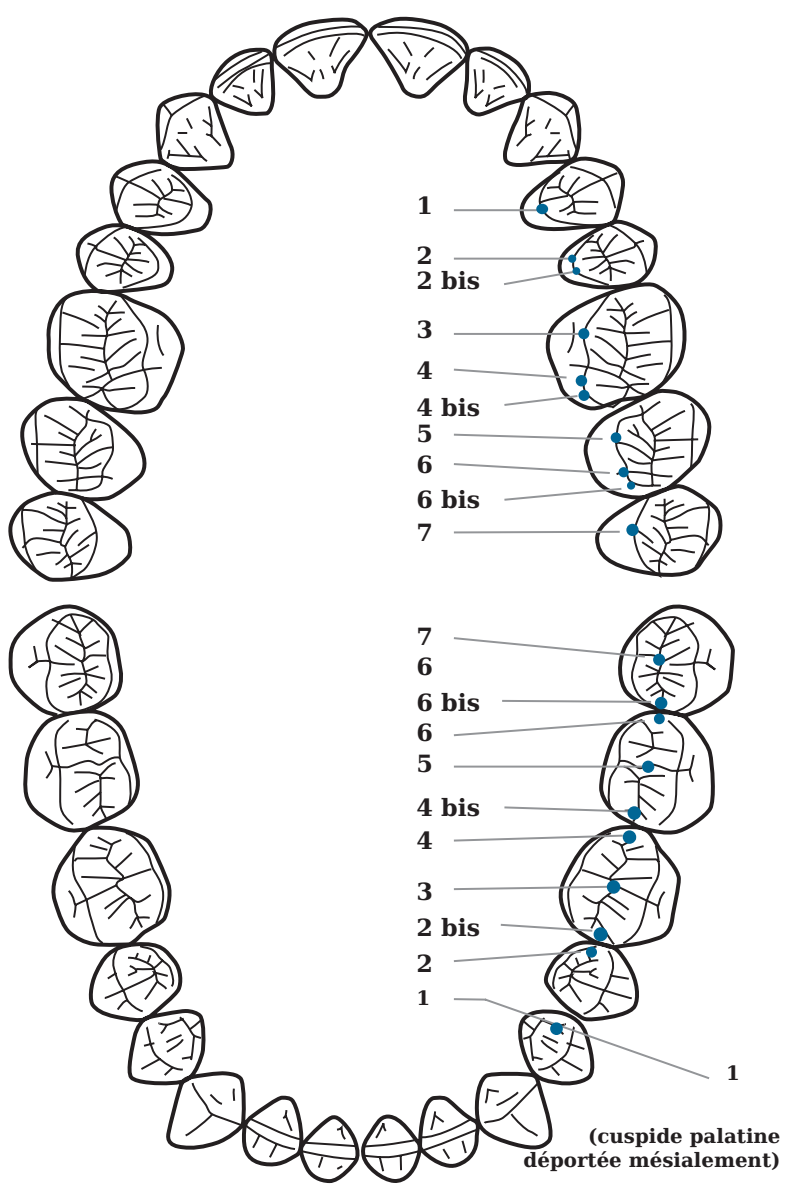

Fig. 1

obtenons les meilleurs renseignements dans le sens transversal. Le tracé des axes des molaires perpendiculaire à leur face occlusale est une aide précieuse. Une petite équerre d'assemblage en fer plat de $10 \mathrm{~mm}$ de large, avec des branches de 5 à $7 \mathrm{~cm}$, facile à trouver dans un magasin de bricolage, devient un excellent instrument de traçage. Le pliage à $90^{\circ}$ d'un plat d'aluminium de $10 \mathrm{~mm}$ de large dans un étau reste une solution facile à réaliser et évite le problème posé par les trous de vissage.

Les différentes cuspides des faces occlusales des molaires ne sont pas à la même hauteur et l'appui direct d'un segment de cette équerre sur ces pointes cuspidiennes n'indiquerait pas avec l'autre segment l'axe véritable de la dent. Il est indispensable en s'appuyant sur la cuspide la plus haute de paralléliser le segment occlusal de l'instrument avec les crêtes marginales, seules références perpendiculaires au grand axe des dents pluricuspidées (figure 2 et 3). Les points $n^{\circ} 3$ ou 5 (figure 1 ) devant normalement se correspondre, il est intéressant de tracer les axes des molaires en passant par ces repères. Si on dispose d'un peu de temps, deux perles de silicone écrasées sur les faces occlusales avec cette équerre en bonne position faciliteront la manœuvre.

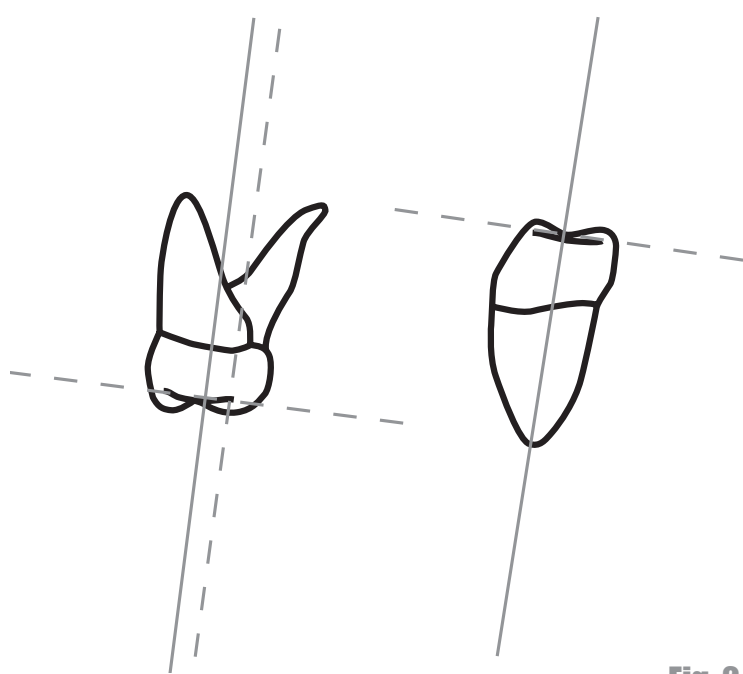

Fig. 2 et 3

Le tracé des axes des molaires sur le dos des modèles (photo 2, lignes 3 et 4), même si une erreur de quelques degrés reste possible, devient beaucoup plus précis à l'aide de cet instrument d'un coût minime. Par la même occasion, en poussant le segment occlusal jusqu'au collet de la dent opposée, il est possible de vérifier la courbe de WILSON en s'appuyant cette fois sur les pointes des cuspides des molaires mandibulaires. Si l'intersection de ce segment avec la gencive opposée se produit en dessous du collet, le diagnostic de linguo-version peut être évoqué.

\subsection{La mesure millimétrique du déficit transversal}

Si maintenant on considère que les contacts inter-arcades des groupes III (figure 1) sont des objectifs à atteindre pour obtenir une « santocclusion » (bulletin $\mathrm{n}^{\circ} 35$ de mars 2008) il est facile de marquer au crayon papier les points $\mathrm{n}^{\circ} 3$ sur les modèles. Les non-concordances sagittale et transversale de ces points entre l'arcade maxillaire et l'arcade mandibulaire en occlusion sont une source possible de 
dysfonctionnement au cours de la mastication et de l'appui nécessaire à une déglutition adulte. Une mesure transversale au pied à coulisse permet, à l'aide d'une soustraction, de quantifier avec précision l'expansion qui sera nécessaire au niveau des premières molaires. Lorsque l'arcade maxillaire est en forme de $\mathrm{V}$ on peut de la même façon mesurer les points 1 sur les premières prémolaires pour connaître l'expansion à réaliser dans la région antérieure.

Enfin ces observations doivent être mises en corrélation avec le photo-montage préconisé par P. VION. Il consiste, après avoir marqué au crayon la suture médiane palatine, les points no 3 ou 5 (et éventuellement 1), à tirer une photocopie de ces modèles posés sur leurs arcades sur la vitre du photocopieur.

Ensuite, sur la photocopie des moulages il faudra situer l'emplacement futur des fonds des tubes molaires et des brackets canins sur les faces vestibulaires. Il suffit alors de positionner des transparents des formes d'arcades les mieux adaptées sur ces photocopies (figure 4). Pour l'arcade maxillaire on veillera à bien faire coïncider l'axe médian avec les points repérant

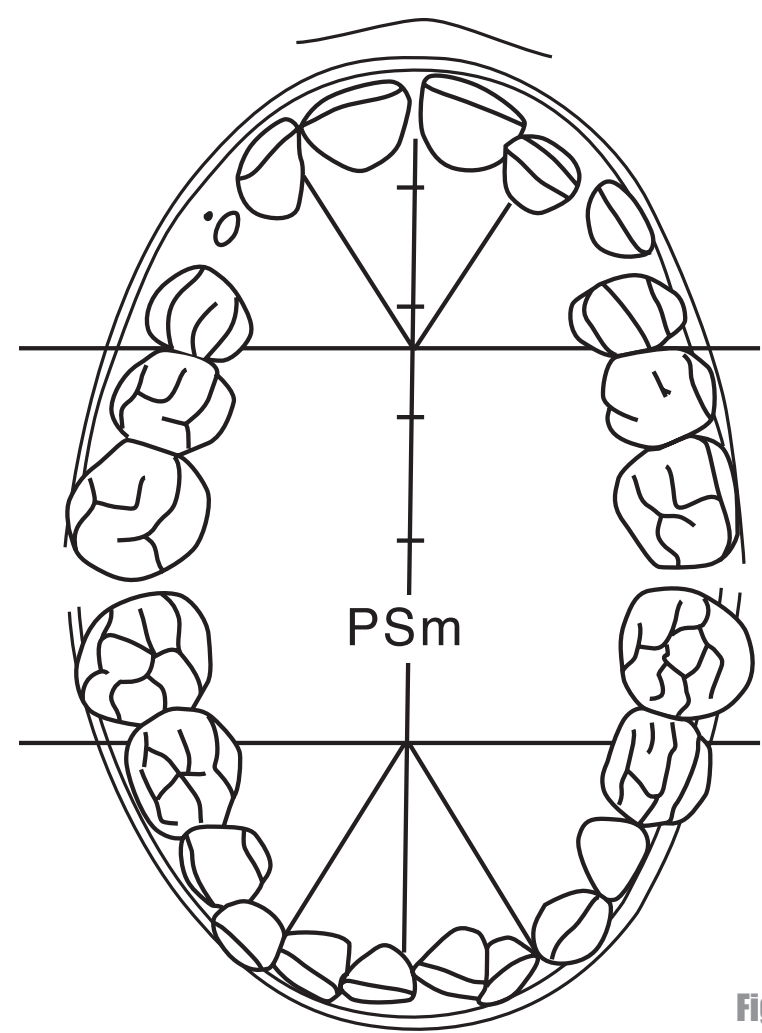

la suture médiane. Pour l'arcade mandibulaire c'estl'axe transversal qui devra être parfaitement parallèle aux faces mésiales des 36 et 46 si ces dents n'ont pas subi de mésio-version (à la suite de l'extraction prématurée d'une 75 ou 85 par exemple).

La forme d'arcade choisie parmi les 5 formes décrites par RICKETTS représente un arc idéal d'une technique vestibulaire. Il doit se placer sur les fonds des attaches scellées sur les premières molaires et les canines qui sont les dents guides de la future arcade corrigée.

Ce photo-montage permet d'abord de vérifier les symétries maxillaires sagittale et transversale. La forme d'arcade doit être choisie de façon que la distance entre les tubes molaires et l'arc corresponde à l'expansion nécessaire obtenue par la soustraction que nous avons effectuée précédemment. Si à ce stade une dissymétrie de l'arcade maxillaire est notée, elle doit être immédiatement identifiée (voir le cas no 7).

\subsection{L'analyse des tracés}

Les quatre grands axes des molaires ont été tracés, l'observation des modèles par la face postérieure permet de distinguer plusieurs cas :

1. La voûte palatine a une forme harmonieuse, les grands axes des molaires sont convergents vers le haut et perpendiculaires à la courbe de WILSON, le résultat de la soustraction entre les mesures maxillaires et mandibulaires des points 3 et 1 est égal à zéro, le photo-montage permet de placer la forme d'arcade choisie exactement sur le fond des attaches vestibulaires, il n'y a aucune correction transversale à effectuer (figure 5) .

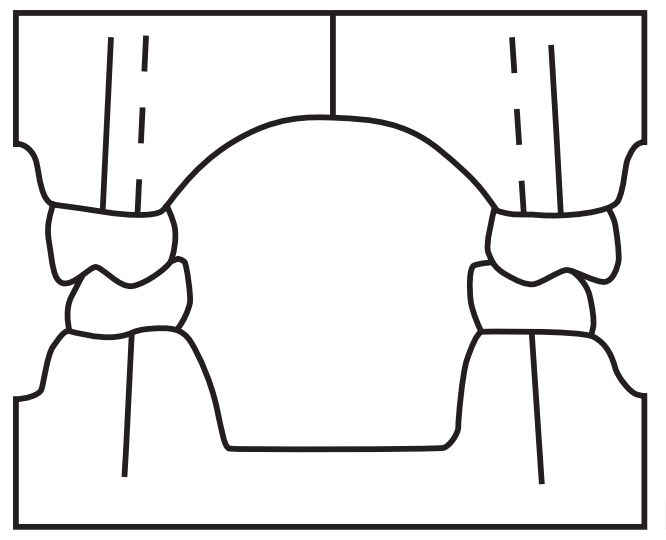


2. La voûte palatine est étroite, les grands axes des molaires sont convergents vers le haut et perpendiculaires à la courbe de WILSON, le résultat de la soustraction est égal à une certaine valeur, cette valeur est retrouvée à l'aide du photo-montage. Il s'agit d'une endomaxillie basale (figure 6). Les rapports molaires transversaux sont altérés d'un ou des deux côtés.

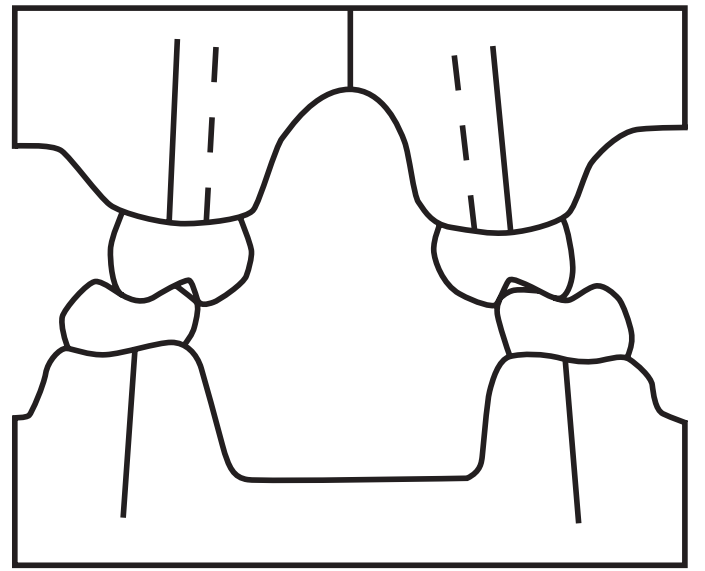

Fig, 6

3. La voûte palatine est étroite, les grands axes des molaires maxillaires sont convergents vers le haut et perpendiculaires à la courbe de WILSON, le résultat de la soustraction est égal à une certaine valeur, cette valeur est retrouvée à l'aide du photo-montage. Les rapports transversaux sont altérés d'un ou des deux côtés, mais les axes des molaires mandibulaires tendent à devenir parallèles et ne sont pas perpendiculaires à la courbe de WILSON. Il s'agit d'une endomaxillie basale accompagnée d'une exoalvéolie mandibulaire (figure 7).

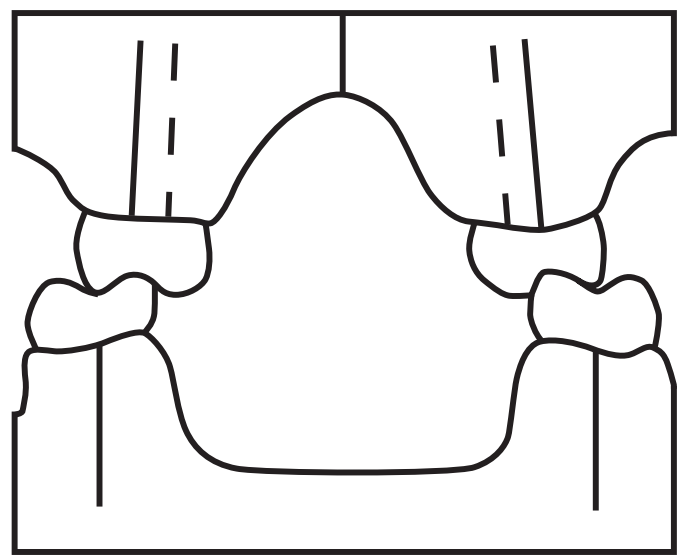

4. La voûte palatine présente une dissymétrie transversale, les grands axes des molaires mandibulaires sont convergents et perpendiculaires à la courbe de WILSON mais non les axes des molaires maxillaires, le résultat de la soustraction est égal à une certaine valeur, cette valeur est retrouvée à l'aide du photo-montage, il s'agit d'une endoalvéolie maxillaire qui peut être uni ou bilatérale. Les rapports molaires transversaux sont altérés. Le photo-montage permettra d'identifier le côté « coupable » avec précision (figure 8).

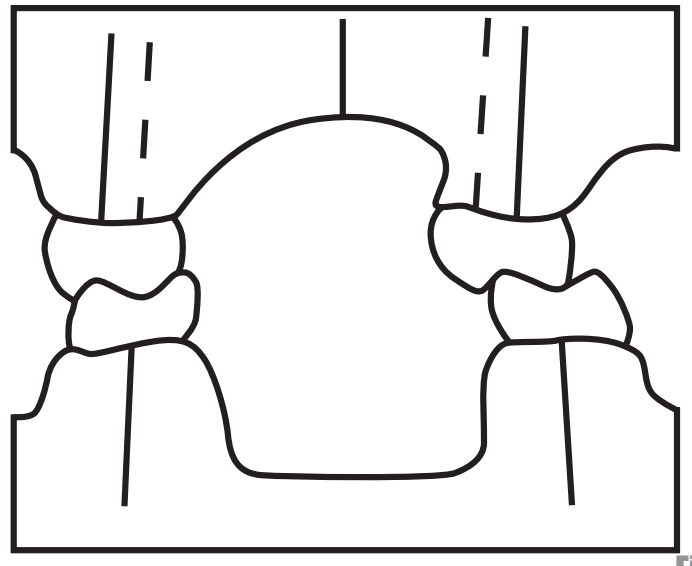

Fig. 8

5. La description est la même que précédemment mais les axes des molaires mandibulaires sont eux aussi trop convergents et non perpendiculaires à la courbe de WILSON. Il s'agit d'endoalvéolies maxillaires et mandibulaires (figure 9).

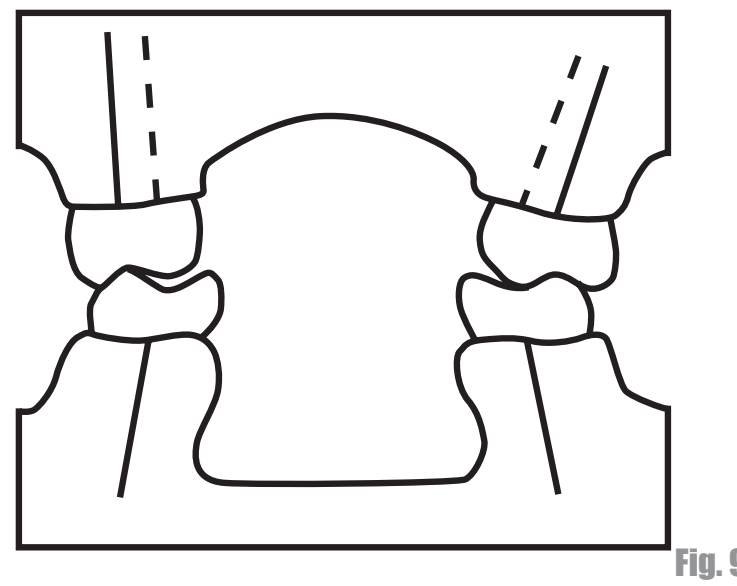

6. La voûte palatine est étroite, les grands axes des molaires ne sont plus perpendiculaires à la courbe de WILSON. Mais les rapports 
molaires transversaux ne sont pas altérés. Le résultat de la soustraction peut être égal à zéro. Il s'agit d'une endomaxillie basale compensée par une exoalvéolie maxillaire et une endoalvéolie mandibulaire (figure 10).

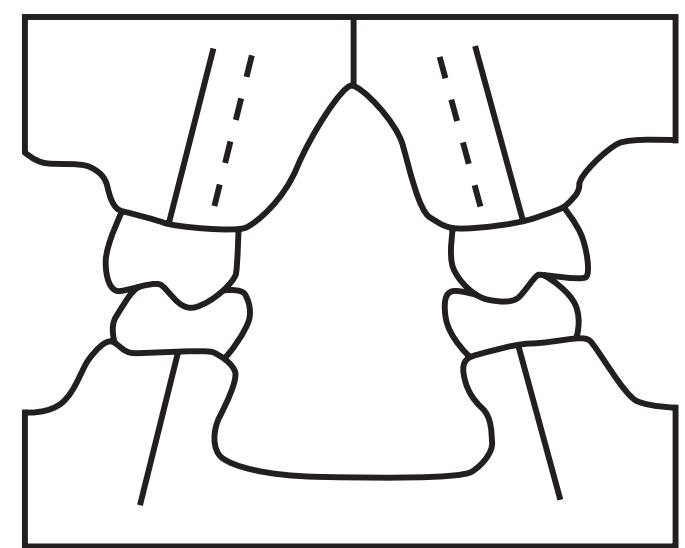

Fig. 10

7. S'il s'agit d'une endomaxillie basale dissymétrique, il faudra en rechercher l'origine à l'aide de documents complémentaires 3D. Si c'est la base du crâne qui est dissymétrique, nous n'avons pas de thérapeutique à proposer. Si c'est seulement un sinus maxillaire, il faut rechercher la cause de la dysfonction ventilatoire de ce côté et essayer d'intervenir s'il est encore temps.

8. Les rapports molaires transversaux peuvent être altérés par excès maxillaire... On peut ainsi continuer à imaginer tous les rapports possibles, tracer les dos des modèles avec la même rigueur et diagnostiquer les cas les plus complexes.

9. Il est difficile de classer la mésiorotation molaire. À première vue, il s'agit d'une dissymétrie sagittale qui accompagne la mésiogression d'un secteur latéral, mais la racine palatine de la molaire maxillaire joue le rôle d'un pivot autour duquel va tourner la dent. Cette rotation de la molaire entraîne aussi une endo-alvéolie. L'analyse de ces cas nécessite l'usage du tracé des dos des modèles et du photomontage. C'est pourquoi il nous paraît intéressant de la classer parmi les pathologies transversales. D'autre part les mécaniques envisagées pour leur traitement peuvent faire partie de la famille des mécaniques à effet transversal.

\subsection{Proposition d'une classification transuersale}

L'activité et la position de repos de la langue et des buccinateurs sont immédiatement vues et comprises grâce à ces tracés des dos des modèles. La complexité de description des nombreux cas ci-dessus évoqués et de toute leurs familles nous incitent à proposer une classification. Pour ne pas la confondre avec la classification des rapports antéro-postérieurs d'ANGLE il ne faut pas utiliser de chiffres mais seulement des lettres. Cette classification doit pouvoir être vérifiée rapidement en examinant la bouche de face, dents en occlusion de convenance. Le vocabulaire anglais étant de règle pour les sciences odontologiques, nous retiendrons les initiales des termes utilisés :

$\mathrm{T}$ pour transversal class,

$\mathrm{U}$ pour up pour maxillaire,

D pour down pour mandibulaire,

I pour in pour lingual ou palatin,

O pour out pour vestibulaire,

B pour basal ou squelettique,

A pour alveolar ou dento-alvéolaire,

$\mathrm{R}$ pour rotation.

Les troisièmes termes ne peuvent être précisés qu'une fois effectués les examens que nous avons décrits mais les deux premiers sont faciles à identifier en bouche.

Ainsi le cas no 2 serait une T-UIB pour classe transversale : Up In Basal, le no 3 une T-UIB, DOA pour classe transversale : Up In Basal et Down Out Alveolar, le no 4 une T-UIA pour classe transversale : Up In Alveolar, le no 5 une T-UIA, DIA pour classe transversale : Up In Alveolar et Down In Alveolar, le no 6 une T-UIBOA, DIA pour classe transversale : Up In Basal Out Alveolar et Down In Alveolar, etc.

Les avantages de cette classification seraient d'abord de nous obliger à décrire précisément le trouble transversal, de communiquer entre nous sans risques d'erreurs et surtout d'en déduire immédiatementlasolution thérapeutiqueadaptée. Un défaut basal ne peut être traité que grâce à une mécanique orthopédique et le plus précocement possible alors qu'un défaut alvéolaire sera traité par une mécanique orthodontique et beaucoup moins sous la dépendance de l'âge et du potentiel de croissance résiduel. À bon diagnostic, bonne prescription! 
Nous étudierons dans le prochain bulletin les mécaniques à effet transversal.

Un défaut transversal peut être identifié très précocement, au cours du dépistage des troubles dentaires ou parodontaux. L'attention requise ne demande que quelques secondes à un praticien qui recherche ces troubles : incisions décalés, articulé molaire croisé, déviation du chemin de fermeture, limitation d'ouverture buccale, douleur ou bruits au niveau des ATM, gêne à la mastication. Notre intervention peut donc être envisagée chez le tout petit. Nous avons traité des troubles transversaux dès l'âge de trois ans à la grande surprise de certains contrôles de sécurité sociale.

Nous distinguerons les mécaniques à effet orthopédique de celles à effet orthodontique bien que ces dernières aient parfois un effet orthopédique associé.

\section{EXEMPLE PRATIQUE D'EXPANSION MAXILLAIRE PAR DISJONGTEUR GHEZ UNE PATIENTE AGÉE DE 8 ANS [SUTURE INTERMAKILLAIRE FERMÉE APRĖS 7 ANS]}

- disjoncteur à expansion uniforme, exemple : articulé inversé bilatéral avec endomaxillie
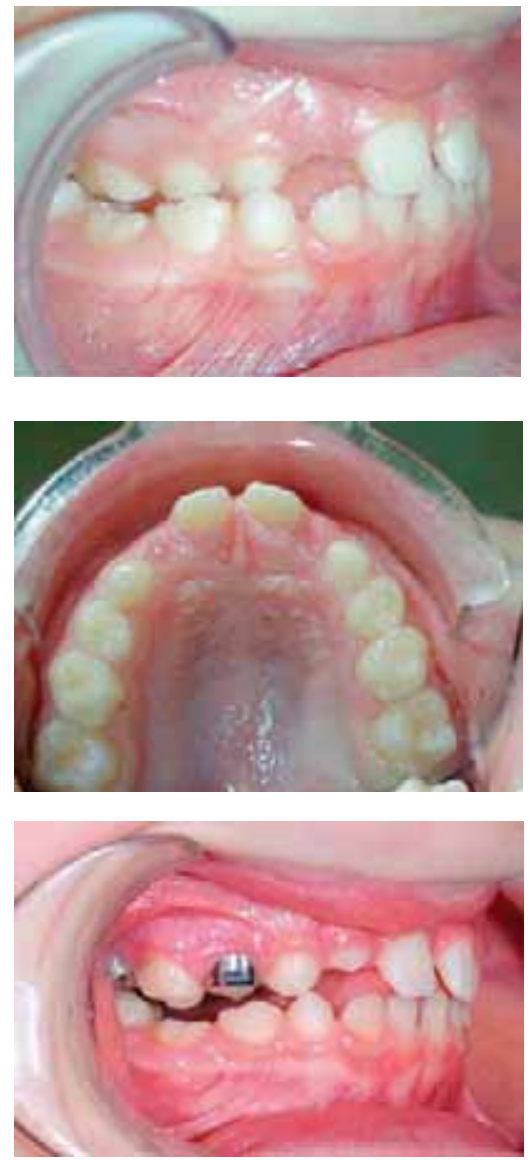
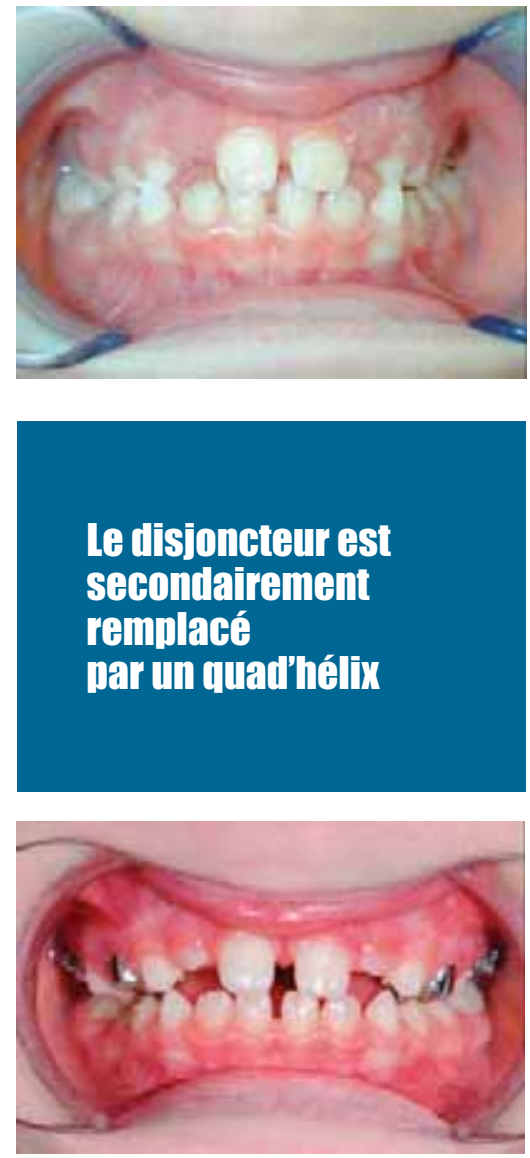
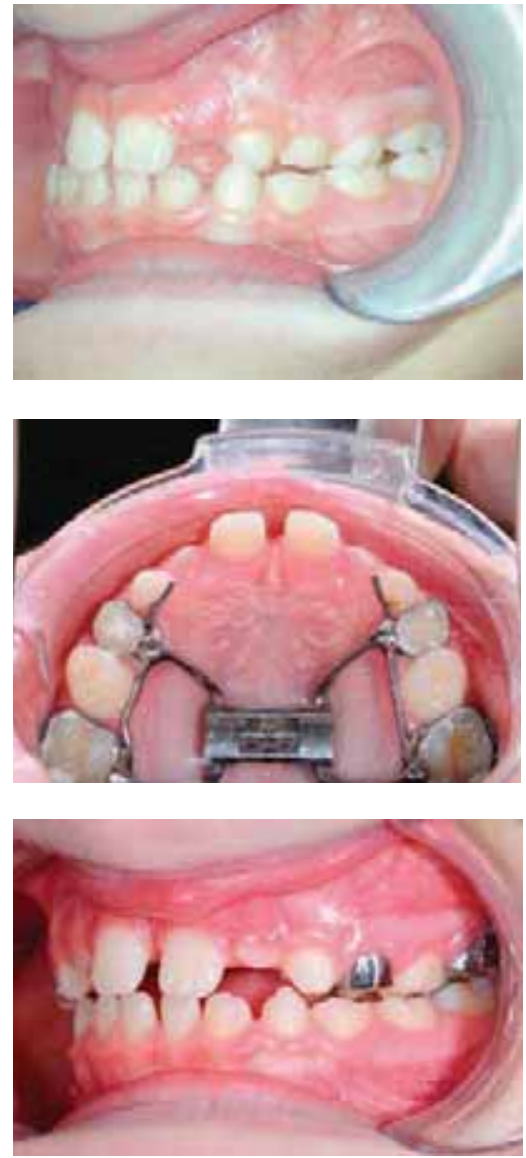

Disjoncteur réalisé en soudant la partie " araignée » sur les bagues des molaires définitives et lactéales, afin d'éviter la version vestibulaire.

26 quarts de tours (un par jour, le soir) sont effectués avec la clef à vérin. L'espacement des incisives centrales supérieures prouve la disjonction de la suture. L'articulé inversé étant plus accentué à droite, les milieux incisifs se recentrent.

Le disjoncteur est secondairement remplacé par un quad'hélix, moins encombrant, pour maintenir le résultat, ce qui permet de commencer la rééducation de la déglutition primaire associée, compte tenu que la «boîte à langue » est plus volumineuse. 